\title{
Long Term Epistemic Actions
}

\author{
Mark-Oliver Casper \\ Cluster of Excellence Image Knowledge Gestaltung, Humboldt University, Berlin \\ Department of Philosophy II, Ruhr-University, Bochum \\ casper@bildakt.de
}

Received 31 August 2016; accepted 10 May 2017.

\begin{abstract}
The enactivist term mental institution was introduced to the situated cognition debate to conceptualize profound coherencies between cognitive processes and institutional settings. This article starts by criticizing the idea of mental institutions since it is frequently said that they are complex epistemic actions. By making explicit what "epistemic action" actually refers to, it becomes apparent that mental institutions cannot be seen as a complex form of such an action. In the second step, the mental institution idea is retained and supported by distinguishing two kinds of epistemic actions: short-term epistemic actions and long-term epistemic actions. Mental institutions cannot be treated as short-term epistemic actions as this kind of action requires computationalist commitments about which enactivists are explicitly critical. However, they can be considered as long-term epistemic actions - as settings which allow the planning and designing of short-term epistemic actions.
\end{abstract}

Keywords: situated cognition; mental institution thesis; epistemic action; pluralistic account of cognitive science.

The idea of mental institutions was introduced to the situated cognition debate by Gallagher and Crisafi. (Gallagher \& Crisafi, 2009; Gallagher, 2011, 2013) The central claim of this idea is that

[c]ertain social institutions (including social practices) are what we might call 'mental institutions ..., in the sense that they are institutions that help us to accomplish certain cognitive processes. Indeed, without them, specific classes of cognitive processes would simply not exist. They are at least enabling conditions, and on the most liberal reading, constitutive of those processes. (Gallagher, 2011, p. 59) 
Enabling conditions are conditions which are systematically and historically necessary for cognitive processes to take place. Or to put it differently: something is an enabling condition if its absence prevents something else occurring (cf. De Jaegher, Di Paolo, \& Gallagher, 2010, p. 443). In this context, legal or scientific procedures are supposed to allow for specific cognitive states and processes. In order to support this line of pragmatist, enactivist reasoning, Gallagher refers to institutions as complex epistemic actions (Gallagher \& Crisafi, 2009, p. 48; Gallagher, 2011, p. 66; Gallagher, 2013, p. 8).

In this paper I want to support the idea of mental institutions but argue against the term "complex epistemic action." Instead of talking of complex epistemic action we need to differentiate between two kinds of such actions: short-term epistemic actions and longterm epistemic actions. In order to talk of epistemic actions at all it is advisable to make explicit to what this term exactly refers. Subsequently, it is possible to elaborate on the question of whether institutions can be a complex form of epistemic actions.

\section{Tetris, Epistemic Actions and Computational Improvement of Limited Cognitive Resources}

Epistemic action is clearly and frequently distinguished from pragmatic action. Pragmatic actions "create physical states which physically advance one towards goals" (Kirsh \& Maglio, 1994, p. 513). In this sense they are actions which are executed to directly achieve a certain end or to solve a problem. Other actions are better understood as serving an epistemic function. This means that such actions do not directly contribute to problem-solving, but they enhance the problem-solving skill of a person. From Kirsh's \& Maglio's point of view, there are physical actions that make mental computation easier, faster and more reliable with regard to solving problems. These are epistemic actions - physical actions performed by a cognitive organism to alter its own computational states by changing the environment in which the epistemic function consists. These changes are said to yield three benefits: (I) reducing space complexity, (II) reducing time complexity, and (III) reducing unreliability (cf. Kirsh \& Maglio, 1994, p. 514).

The efficiency of cognitive problem-solving increases or decreases in relation to how efficiently cognitive organisms can exploit external informational settings. By altering the way in which a problem can be approached, limited cognitive resources can be improved. To put it differently: "Epistemic actions are actions designed to change the input to an agent's information-processing system" (Kirsh \& Maglio, 1994, p. 541). However, it is also important to note that "a sequence of actions may . . . return the physical world to its starting state and significantly alter the ... [cognitive agent's] informational state" (Kirsh $\&$ Maglio, 1994, p. 515). This is crucial to mention because the environmental change is not necessarily a constant one.

The notion of epistemic action thus understood highlights the close interaction between a cognitive agent and the surrounding setting which allows the cognitive-computational burden to be offloaded to environmental structures. The context of cognition affects cognitive 
performances because computation is said to happen not merely inside the cognitive agent. This reasoning is in line with the extended mind thesis in terms of cognition as something that is organism-centered and functionally distributed. In Clark's words:

Concerning the process of recruitment [of cognitive technology or environmental structures], it is indeed the biological brain ... that is in the driver's seat. That is to say, it is indeed some neurally based process of recruitment that ... turns out to be so pointedly unbiased regarding the use of inner versus outer circuits, storage and operations. . . What this suggests . . is that in rejecting the vision of human cognitive processing as organism bound, we should not feel forced to deny that it is ... organism centered. (Clark, 2011, p. 122f)

The strong theoretical connections between the notion of epistemic action and the extended mind thesis becomes even more apparent if an example of an epistemic action is presented. A prominent example of an epistemic action is a certain kind of performance by players of the video game Tetris. The aim of this game is to align digital blocks of various shapes in a way that no gaps are left between them. These blocks fall from the top to the bottom of the display, where they accumulate. However, if a row of blocks is arranged properly (without gaps) then it vanishes from the display and the player can continue to place blocks and collect points until the blocks reach to the very top of the display. In order to arrange blocks properly, players are able to move the blocks to the right and left and rotate them. Kirsh and Maglio observed that the better the player, the more redundant spins and moves are conducted while playing. These additional movements of blocks first take them farther from their best slot. Prima facie, these movements seem unnecessary or even disadvantageous, but actually they enhance the player's Tetris skills. This example is supposed to show that players get better while doing redundant spins of blocks because by doing so they acquire information about the block such as its form and where it should be placed. Such information would be less efficiently achieved by rotating blocks mentally or planning the next move merely "in the head."

So, epistemic action is defined and used in contexts in which cognitive processes are commonly understood as some kind of mental computing. Kirsh and Maglio (1994) claim that the chief novelty of the use of epistemic action "lies in allowing individual functional units inside the agent to be in closed-loop interaction with the outside world" (p. 543).

Another example of this kind of epistemic action is introduced by Hutchins, who is interested in understanding how cognitive capacities such as remembering come about. As an "ecological psychologist" he does not choose individual organisms or parts of organisms as the basic unit of his analyses. Instead, in order to study cognitive phenomena, he pays attention to interaction patterns between organisms and environmental structures. One example of this is his study of the design of an airplane's cockpit that enables pilots to track various important physical quantities while flying a plane (Hutchins, 1995). In a nutshell, Hutchins refers to as a distributed cognitive system the placement of instruments within the cockpit, the actual manipulability of controls, and the interaction of the pilots in the cockpit. The systematic entanglement of all of these aspects is supposed to constitute the ability to detect and to remember, for example, how fast the plane is flying. One interesting aspect of 
this example is that it seems to be neutral with regard to computationalist commitments. Computationalists may assume that the "ecological" focus of cognitive scientists like Hutchins does not necessarily rule out the idea that cognition is basically dependent on an interplay of internally and externally realized computational processes. However, computationalists may react to Hutchins' claims: he proposes a practical-ecological approach to explaining cognitive skills and this approach seems to stay silent about the extended mind thesis and the role of "mental computing" in cognitive science (cf. Hutchins, 2010).

But even if Kirsh \& Maglio on one hand and Hutchins on the other pursue different theoretical approaches, they nevertheless share the idea that the contexts of (individual) cognitive performances affect them not because of contingent causal interrelations but because they strongly and mutually assume each other. However, both examples can be considered as basically referring to short-term epistemic actions. Following Kirsh \& Maglio to an extent, I would like to introduce the notion of short-term epistemic action by defining it as physical actions which serve an epistemic rather than a pragmatic function since such actions aim to tune interactions between agent(s) and settings in a way that achieves a strong mutual fit and fluent problem-solving for single and concrete tasks in specifiable local conditions. While playing Tetris, redundant moves and rotations of blocks is a cognitive strategy that yields its benefit only gradually. This strategy can be employed for every new approaching block. Similarly, the cockpit is designed to keep track of physical entities during a specific flight. In the context of these tasks (playing a Tetris game or flying a plane from A to B) one can talk of short-term epistemic actions with regard to the starting and ending task. Short-term epistemic actions help to solve such tasks case by case. This aspect of short-term epistemic actions helps to distinguish them from long-term epistemic actions and to show that institutions or institutional settings can only allow for actions of the latter kind.

\section{Grounding Assumptions for the Idea of Mental Institutions}

To make solid sense of the idea of mental institutions it is advisable to show on which assumptions it is based. Two further assumptions are pivotal: (I) the parity principle as posed by Clark is only partially sustainable; (II) if the parity principle is partially compromised then it is advisable to consider alternative conditions to replace those involved by the parity principle and which, if satisfied, allow a certain state and process to be called "cognitive" regardless of where these states and processes take place.

Concerning assumption one: The parity principle says that cognitive processes are not all in the head when a physical resource outside of a cognitive organism meets three conditions: (1) such a resource is reliably available and typically invoked when dealing with certain cognitive tasks; (2) the resource carries information and this information is automatically endorsed when retrieved; (3) the information contained in the resource is quickly and easily accessible. (Gallagher \& Crisafi, 2009, p. 46) The claim being made is that if these conditions are satisfied, external props can be part of cognitive computation as computational processes are then treated as (partially) off-loaded on to the environment. 
But all three conditions are rejected by Gallagher and Crisafi as each of the criteria involves matters of degree. Some instrument might be able to allow a specific cognitive task or problem to be solved, but it is not easily accessible. A prominent example in this case is the problem of evaluating a certain state of affairs from a legal perspective by consulting instruments and rules of the legal system. The legal rules and processes that need to be applied in order to legally evaluate a state of affairs properly are not easily accessible (condition one) but indeed lead to cognitive performance (judging) that could not be conducted without it. Additionally, the second condition is under suspicion. The condition of automatic endorsement seems to be a little too restrictive as information can play a role in cognitive processes without being accepted as true or rejected as not applicable (cf. Gallagher, 2013, p. 6). For Gallagher, this examination results in the claim that neither "a violation of these [three] criteria, nor any lack of parity with internal mental states, should disqualify such processes from being considered cognitive if they are processes to which the human organism is linked in the right way, that is, 'in a two-way interaction, creating a coupled system that can be seen as a cognitive system in its own right"' (Gallagher, 2013, p. 6). But even if all three conditions are dismissed by Gallagher, it does not involve a rejection of the parity principle altogether. The critique against the aforementioned conditions pursues not a rejection but a more liberal reading of the parity principle - a reading that is supposed to enable us to consider more entities as a proper part of a cognitive system. This idea is justified by

taking up a new conception of the mind, and a new understanding of how the brain works. Here I want to take seriously the idea that the term 'extended' goes along with . . . other terms: 'embodied', 'embedded', and 'enactive'. We need to conceive of the brain, not as the place where all the mental processing and representing happens, but as part of a larger ... system; and we need to conceive the mind, not on a functionalist interpretation (pace Clark, Wheeler, and many others), but as enactively generated in the specific interactions of organism-environment ... The mind, not as a collection of propositional attitudes, representations, mental states, etc., all of which have intrinsic or non-derived content, but the mind as an enactive and emotionally embedded engagement with the world through which we solve problems, control behavior, understand, judge, explain, and generally do certain kinds of things. On this conception, the mind is constituted primarily by just such activities. (Gallagher, 2011, p. 65)

The objective to conflate the different approaches of the situated cognition debate (extended, embodied, enacted, embedded mind) spurs Gallagher to apply the parity principle in a way that broadens the spectrum of entities which can be considered in theories of cognition. A liberal reading of the parity principle results in the rejection of Clark's conditions that were supposed to restrict the scope of entities that proponents of the extended mind need to deal with when talking about what constitutes cognition.

Concerning assumption two: To maintain that something constitutes something else is a contentious issue. Extended Mind proponents, for example, hold that cognition is sometimes constituted by non-biological and non-bodily entities. On the other hand, internal computationalists claim that - in terms of cognitive states and processes - the brain is the only thing worth considering in cognitive science since only the brain is able to generate a 
special kind of content. There are two crucial claims in this context: (1) the "cognitive processes we find in the real world all happen to be brain bound" (Adams \& Aizawa, 2001, p. 46); (2) the "essential condition on the cognitive is that cognitive states must involve intrinsic, non-derived content” (Adams \& Aizawa, 2001, p. 48).

However, it is more productive to focus on general aspects that, if considered, can help to show when something counts as a proper part of the cognitive system. These aspects help to structure the "constitution talk" in philosophy of mind and cognitive science. De Jaegher, Di Paolo and Gallagher propose that circumstances in which something occurs (or comes into existence) are well analyzed by considering contextual factors, enabling conditions and constitutive elements. Let " $\mathrm{X}$ " be something in which researchers are interested, then " $\mathrm{F}$ is a contextual factor if variations in F produce variations of $\mathrm{X}, \mathrm{C}$ is an enabling condition if the absence of $\mathrm{C}$ prevents $\mathrm{X}$ from occurring and $\mathrm{P}$ is a constitutive element if $\mathrm{P}$ is part of the process that produce X" (De Jaegher, Di Paolo, \& Gallagher, 2010, p. 443).

This means that contextual factors have an effect on X: enabling conditions not only affect $\mathrm{X}$ but are systematically or historically necessary for $\mathrm{X}$ to take place and constitutive elements must be present at the same time as X since they are part of the phenomenon. In this context, enabling conditions are also contextual factors as they influence $\mathrm{X}$; constitutive elements are also enabling conditions since they allow $\mathrm{X}$ to occur. The authors add that "[w]hat exact role . . . an element plays in X depends on how one chooses to describe and observe X” (De Jaegher, Di Paolo, \& Gallagher, 2010, p. 443).

If we take Gallagher's enactivist conception of the mind into account that mainly states that our practical involvements with institutional settings and practices are pivotal for certain cognitive states and processes to arise, and if we combine this conception with the differentiation of contextual, enabling and constitutive elements, then the basic claim that institutions can enable or even constitute cognitive states and processes becomes reasonable. However, there is still a problem with his liberal reading of the parity principle.

\section{Enactivists and Hutchins' Promiscuous Cockpit}

Basing the idea of mental institutions on a liberal form of the parity principle is difficult to justify because the parity principle is coupled to the claim that cognition is organismcentered. Moreover, the parity principle usually works in a theoretical context which endorses a computationalist picture of the mind.

We need to concede further that if extended mind functionalists endorse the claim that cognition is organism-centered, the three conditions which were added by Clark (reliable Availability, automatic Endorsement, easy Access) become more plausible than they might seem at first glance. The problem with Gallagher's critique of these conditions is that criticizing the parity principle and cutting it from its functionalist and computationalist context to use it as grounds for the idea of mental institutions actually means dissolving the parity principle altogether. In such a case we would no longer be dealing with the parity principle. If this is correct, to address this principle as Gallagher does is a redundant effort. 
This also seems true when we look at the example of a mental institution. As an example of a mental institution, the legal system makes clear that certain cognitive states and processes should be treated as supervening on a shared practice. To base cognition on shared practices only fits into a pragmatist and enactivist account that is not prima facie able to accommodate the parity principle since it deals with cognition as something computational as well as organism-centered.

Additionally, Gallagher interprets the extended mind thesis as hinging on a further concept of the mind he is not willing to accept:

This concept "focuses on specific kinds of mental states (belief, or belief embedded in memory ...), explicates the three criteria that seem to apply to such mental states, and then generalizes the criteria to apply to all cognitive processes. The controlling conception of the mind that guides this analysis, then, is that the mind is constituted by beliefs, desires, and other propositional attitudes and for Clark and other extended mind theorists . . .), by representations and informational states as well. But neither the standard belief-desire psychology nor these criteria necessarily apply to all cognition, especially if one thinks of cognition in dynamic terms of enactive cognitive processes and activities, e.g., problem-solving, interpreting, judging, rather than in terms of mental states or static content." (Gallagher 2013, p. 5)

This quote makes it rather urgent to put the parity principle aside instead of proposing a liberal reading of it.

A similar critique may be given against Gallagher's use of the notion epistemic action. The original paper of Kirsh \& Maglio takes a rather functionalist and computationalist view. Hence, the concept of epistemic action seems to presuppose the acceptance of a partly-internalist, computationalist, functionalist account. Enactivist, computationalist and functionalist perspectives are not easily reconciled, and the notion of epistemic action is therefore not straightforwardly employable by enactivists.

It might be reasonable to say that if Hutchins' example counts as an example of a shortterm epistemic action, then institutional practices may also count as a short-term epistemic action because Hutchins - like Gallagher-does not rely on computational processes to make his point. A proper answer to this question is that Gallagher is explicitly not neutral when it comes to computationalism. On that note, Hutchins is not as theoretically transparent as Gallagher and is therefore theoretically promiscuous. Hutchins' cockpit could be treated as an example of a short-term epistemic action as Kirsh, Maglio, or Clark just need to add that the mechanisms behind such a mutual fit between cockpit and pilot (as stressed in Hutchins example) are based on distributed computational processes.

If Gallagher wants to use Hutchins' cockpit as an example of an epistemic action, problems also arise. Using the cockpit example as an epistemic action from an enactivist perspective is problematic since enactivists could not until now explain why the improvement of cognitive behavior elicited by environmental modifications comes about. They do not provide the justifying consequential commitments, i.e. computational ones. Enactivists can only talk of some kind of feedback loop to describe what is going on in Hutchins' cockpit, but feedback loops do not seem to be the same as epistemic actions. They are especially different if one 
considers that feedback loops can also refer to things like financial systems or elevators, which are non-cognitive entities. Short-term epistemic actions are totally organism-centered and therefore refer exclusively to cognitive organisms. Secondly, Gallagher's idea of mental institutions does not meet the definition of short-term epistemic actions as they can only be found in specifiable conditions and be employed for specific tasks. This means that they are either not epistemic actions at all, or they constitute a further kind of such actions. This aspect is pivotal and needs to be examined in greater detail in the next section.

\section{Achieving Mutual Fit on Purpose}

In order to talk of mental institutions as a kind of epistemic action, three challenges need to be accepted. This kind of epistemic action (i) needs to be about performances of cognitive agents, but (ii) these performances do not deal with concrete or single tasks and (iii) they are not organism-centered. In a nutshell, for this to work enactivists should consider the difference between short-term epistemic action and long-term epistemic action.

The first kind of action is a physical action which aims to tune interactions between agent(s) and settings in such a way that a strong mutual fit and fluent problem-solving for single and concrete tasks in specifiable local conditions are achieved. The second kind of action incorporates performances whose aim is to plan, design and put short-term epistemic actions to use. Such performances consist in analyzing which problems or tasks need to be systematically tackled and how they can be structurally approached.

Such performances turn out to be particularly interesting. They enable short-term epistemic actions by developing surroundings in which they can occur. Again, take Hutchins' cockpit. In order to have a cockpit one mostly assumes a company - an airline - that needs planes, cockpits, and pilots as well as the best mutual fit between them to secure safe and reliable task performance like starting, flying and landing airplanes. One might also be able to say that in order to play Tetris you need a Game Boy that was developed by a company for electronic entertainment. From this perspective, a long-term epistemic action can easily be about performances of cognitive agents - it is not about elevators but about how cognitive agents achieve their assigned tasks. So, the first condition mentioned above seems to be easily satisfied.

The second condition is also possible to meet. Long-term epistemic actions are not about solving concrete single tasks like flying airplane $\mathrm{X}$ from $\mathrm{A}$ to $\mathrm{B}$ at time $\mathrm{t}$, but about how to deal with every possible future flight. Such actions are about classes of tasks and how they relate to other tasks which also need to be done in order to complete the original one.

This picture allows for the following analogy: given that cognition equates to flying an airplane, then internal computationalists are interested in the turbines of the plane since they contribute the power needed for take-off. Extended mind functionalists focus on the whole plane, thus allowing propulsive force, uplift and steering to be correlated. On the other hand, some enactivists would take this all into account and ask further about the development of the plane, the training of the pilot and the permits needed to fly the plane. With this analogy 
in mind, long-term epistemic actions can be defined as a systematically pursued development of an environmental design that is supposed to achieve the best mutual fit between the cognitive agent and the setting in order to deal with specific classes of tasks.

One might conceive that this way of talking about epistemic actions does not exclude computationalism at all. So-called long-term epistemic actions are just short-term epistemic actions with the goal of producing other short-term epistemic actions; if this is the case, we can go back to just talking of epistemic actions and insist on specific commitments that justify using this notion exclusively in the computationalist framework. However, this is simply not the whole story concerning long-term epistemic actions since there is another important question on the table: Are long-term epistemic actions organism-centered?

They are clearly not. The first point to mention on that score is that the problems or tasks which are tackled by long-term epistemic actions are not directly present in the very situation of analyzing and planning how to deal with them. This is a crucial difference between the close interaction of agent and environment described by Kirsh \& Maglio and the interaction referred to here.

A second point stresses the fact that long-term epistemic actions induce permanent changes as whole settings are implemented, rebuilt, modified or amended. This runs contrary to the claim of Kirsh \& Maglio that an epistemic action may consist of "a sequence of actions [that] may ... return the physical world to its starting state and significantly alter the ... [cognitive agent's] informational state" (Kirsh \& Maglio, 1994, p. 515). This "back to the start" condition is plausible for short-term epistemic actions but not for longterm ones since they need to allow for a whole setting that in turn allows cognitive agents to change and then to return a piece of the physical state of that setting to its starting state in order to deal with an assigned task.

The third point to highlight is that long-term epistemic actions are purposely performed to enable what can be called "short-term epistemic actions." If short-term epistemic actions can at least sometimes partly constitute cognitive states and processes, then long-term epistemic actions are cognition producers - they are conducted with cognition in mind (cf. Gallagher \& Crisafi, 2009, p. 49). Short-term epistemic actions are usually not conducted in the same way as they hinge on cognitive off-loading, which is governed by a special principle called the principle of ecological assembly. According to this principle, the cognitive organism "tends to recruit, on the spot, whatever mix of problem-solving resources will yield an acceptable result with a minimum of effort" (Clark, 2001, p. 13). This recruitment is not explicitly thought through or even known by the agent or organism that carries out actions based on this principle. On the other hand, long-term epistemic actions are not possible if it is not clear what needs to be planned or designed.

By taking these three points into account we reach the conclusion that long-term epistemic actions are not organism-centered because they are not conducted in the situations or environments they structure. They are not adequately depicted as a close and utterly efficient or fast interaction between agent and setting. Furthermore, they do not meet the "back to 
start" condition we find with short-term epistemic actions and they are not just "on the spot" and automatically employed "mechanisms" of ecological assembly. Hence, the actions referred to here as "long-term epistemic actions" do not satisfy various aspects of short-term epistemic actions.

To clarify, there might be short-term epistemic actions which influence other short-term epistemic actions, and such influences might improve cognitive performance without any "cognitive meta-level" or long-term epistemic action. However, short-term epistemic actions just do not seem to always develop so blindly; instead they are explicitly designed and deliberately put to use. The "principle of ecological assembly" gets indirectly exploited by long-term epistemic actions. This difference between two kinds of epistemic actions also accounts for the two different approaches to explaining how cognitive states and processes arise: the computationalist approach and the enactivist approach. With this all said, I claim that mental institutions cannot be presented as a complex epistemic action. One reason for such a claim is that it is simply not intelligible what the difference is between epistemic actions and complex epistemic actions (or "mental institutions"). My assumption here is that there has been no effort to tackle such questions because proponents of the mental institution thesis do not see the necessity. Complex epistemic actions are epistemic actions after all, but not all epistemic actions are mental institutions. However, the basic idea of what a mental institution does or what role it plays for cognitive processes is theoretically better accounted for if the difference between short- and long-term epistemic actions is acknowledged. Moreover, the notion of long-term epistemic action puts situated cognition researchers who are in favor of enactivism in a position to keep their research running without getting bogged down by the question of how to deal with other situated cognition theories and their results. The suggested conceptual distinction separates the two levels of cognitive science research from the philosophical perspective.

\section{Levels and Accounts}

The suggested differentiation between short and long-term epistemic actions is not only theoretically justified. It also nicely more generally reflects the varying levels of explanations and differently pursued accounts in the situated cognition debate. The functionalist and computationalist account of the extended mind thesis as well as the following claims of Kirsh and Maglio, for example, pursue a computational theory of cognition. This engenders shortterm epistemic actions and accompanied principles. Furthermore, they are still able to work closely with neuroscientific disciplines as these are also based on computation. However, they seem to lose track of other circumstances in which such actions are embedded.

On the other hand, enactivists - such as proponents of the mental institution thesis - seem to deem it inessential to theoretically account for what happens with individual brains in certain situations when considering the problem of how to achieve a general theory of cognition. Enactivists highlight whole practical settings and how they enable specific 
interaction formats since biological cognitive agents need to be acquainted with these settings in order to acquire reliable cognitive abilities. This approach is able to consider a state of affairs in terms of development and execution of cognitive skills that other frameworks cannot adequately account for.

However, both approaches do not seem to be easily reconciled, but if the idea of a pluralistic account of cognitive science really is about something then it is about setting aside the problems that might arise when two different approaches are concomitantly applied in order to explain a certain phenomenon and to wait for these problems to be resolved as research progresses. In this way, cognitive science does not just work on one huge system that needs to cover all cognitive phenomena (there is not even a profound agreement on how many of these phenomena exist, or which are the most important ones). This may provide an explorative stance which, if deliberately taken, relies on the expectation of theoretical synergies arising at some point that we cannot yet anticipate. Instead of preventing researchers from elaborating on ideas, philosophy and science should facilitate research and enact at least $a$ conceptual landscape which is capable of accommodating different approaches. The differentiation of short- and long-term epistemic actions allows for just that.

\section{Summary}

This article elaborated on the enactivist idea of mental institutions. Proponents of this idea claim that institutional settings allow specific cognitive states and processes to arise. It is said that they do so because they are complex epistemic actions, but epistemic actions are physical actions that make mental computation easier, faster and more reliable by cognitive offloading and self-induced changes of environmental setups. This is one reason that neither the original notion of epistemic action nor a complex form of it can be used to support the idea of mental institutions.

It has been claimed that the idea of mental institutions can be supported by distinguishing two kinds of epistemic action: short- and long-term. Short-term epistemic actions depend upon the idea that cognitive processes are distributable computational processes and the idea of organism-centered cognition. Long-term epistemic actions are actions which plan, design and put short-term epistemic actions to use. How exactly this is done is an issue for further debate. The objective of this article is just to account for a reasonable and strong difference between the notion of epistemic action as coined by Kirsh \& Maglio and another version of this notion which supports the thesis of mental institutions at a basic level. This has been done by showing that long-term epistemic actions do not meet several conditions necessary for something being treated as a short-term epistemic action. 


\section{References}

Adams, F., \& Aizawa, K. (2001). The bounds of cognition, Philosophical Psychology, 14(1), 43-64.

Clark, A. (2011). Supersizing the mind: Embodiment, action, and cognitive extension. Oxford, UK: Oxford University Press.

De Jaegher H., Di Paolo E. \& Gallagher, S. (2010). Can social interaction constitute social cognition? Trends in Cognitive Science, 14(10), 441-447.

Gallagher, S. (2011). The overextended mind. Versus: Quaderni du Studi Semiotici, 112-113, 57-68.

Gallagher, S. (2013). The socially extended mind. Cognitive Systems Research, 25-26. 4-12.

Gallagher S., \& Crisafi, A. (2009). Mental institutions, Topoi, 28(1), 45-51.

Hutchins, E. (2010). Cognitive ecology, Topics in Cognitive Science, 2(4), 705-715.

Hutchins, E. (1995). How a cockpit remembers its speed, Cognitive Science, 19, 265-288.

Kirsh, D., \& Maglio, P. (1994). Distinguishing epistemic from pragmatic action. Cognitive Science, $18,513-549$. 